\title{
Rechtsgeschichte
}

\section{Martin Schennach}

\section{Mit weinenden Augen und flehenden Händen}


wegung finden. Auch eine kurze Darstellung des Inhalts von Melanchthons Gutachten zu den "Zwölf Artikeln « wäre an Ort und Stelle seiner Erörterung hilfreich gewesen; sie entdeckt man erst später - in einem Abschnitt über die Rezeption des römischen Rechtes in den Beschwerden der Bauern (IO9).

Spannend hätte Deflers Auseinandersetzung mit Kischs Position zu Melanchthons EpieikeiaAequitas-Lehre werden können. Ihr zufolge wird Kisch nämlich mit seiner Ansicht, bei Melanchthon sei »die Milderung der Gesetze nur das Ergebnis einer ganz snormalen interpretatio legum bzw. der Auslegung des Moralgesetzes « (I64), Melanchthon nicht gerecht. Kisch unterstelle diesem eine Verwechslung von Billigkeit (epieikeia) und bloßer Gesetzesauslegung. Nur führt Deflers leider ihre abweichende Position nicht genauer aus und gibt auch keine weiteren Textstellen von Melanchthon an, die gegen Kischs plausible Deutung sprächen.

Überhaupt sind Melanchthons Reden oftmals nur der Anknüpfungspunkt für die Schil- derung der den Kontext bildenden geschichtlichen Ereignisse; beispielsweise dient die Oratio de dignitate legum als Auftakt zur Darstellung der Auseinandersetzungen mit den Osmanen (I49, I 50). Dem an Melanchthon interessierten Leser wäre mit einer Konzentration auf $\mathrm{Me}-$ lanchthons Texte zu Lasten kürzerer Hintergrundinformationen besser gedient, zumal Kisch in seinem schon erwähnten Buch etwa im Kapitel über Melanchthons "Abkehr vom mosaischen Recht « ein gutes Beispiel für genaue Arbeit am Text geliefert hat und korrekt zitiert, übrigens auch mit exakten Seitenangaben, die man in der vorliegenden Arbeit häufiger vermisst.

So hat die Beschäftigung mit Melanchthon diesen sicher weiter aus Luthers Schatten heraustreten lassen. Aus dem Schatten des Referenzwerks über »Melanchthons Rechts- und Soziallehre" hat sich die neue Untersuchung indes nicht lösen können.

\section{Mit weinenden Augen und flehenden Händen*}

Nein, so muss man sich nicht etwa die Reaktion des Rezensenten bei der Lektüre vorstellen, im Gegenteil. Das gedruckte Resultat zweier in den Jahren 1999 und 2000 am italienisch-deutschen Historischen Institut in Trient abgehaltenen Tagungen zu Supplikationen, dessen italienisches Pendant bereits 2002 erschienen ist, liegt nun endlich auch in deutscher Sprache vor. Weinende Augen und flehende Hände gehören vielmehr zur "Supplikation « (Bitt- und
Beschwerdebrief), die als Textsorte während des Untersuchungszeitraumes (I4.-I8. Jh.) regelmäßig (aber nicht durchgehend) standardisiert war und als solche in frühneuzeitlichen »Briefstellern « thematisiert und häufig von professionellen Schreibern abgefasst wurde. Nicht nur inhaltlich empfahl es sich, in Supplikationen möglichst den Erwartungen der angerufenen Obrigkeit zu entsprechen - dies musste auch verbal zum Ausdruck gebracht werden. Man

\footnotetext{
* Cecilia Nubola, Andreas

WÜrgler (Hg.), Bittschriften und Gravamina. Politik, Verwaltung und Justiz in Europa (I4.- I 8. Jahrhundert), (Schriften des Italienisch-Deutschen Historischen Instituts in Trient, Bd. 19), Berlin: Duncker \& Humblot 2005, 458 S., ISBN 3-428-I I 849-9
} 
bat »untertänigst « und »kniefälligst ", um (im besten Fall) eine "gnädigste « und somit gewogene Resolution herbeizuführen.

Konsens dürfte inzwischen darüber herrschen, dass dem Kommunikationsmedium »Supplikation « - ein Musterbeispiel für Massenschriftgut, das einen beträchtlichen Teil des Regierungs- und Verwaltungshandelns auslöste - eine wesentliche Rolle bei der Konstituierung und Ausübung spätmittelalterlicher und frühneuzeitlicher Herrschaft zukam. Durchaus umstritten hingegen erscheint das postulierte Ende der Supplik zur Zeit der Französischen Revolution, als nicht nur der Begriff »scheinbar schlagartig aus dem Wortschatz verschwand « (2I), sondern auch inhaltlich in der "Petition " des frühen Konstitutionalismus aufging. Dabei ist der Terminus in Tirol durchaus (wenngleich mit deutlich abnehmender Frequenz) in der ersten Hälfte des I9. Jahrhunderts belegt, und der semantische Gehalt der »Petition « ist bei weitem restringierter als das umfassendere Phänomen der Supplikation. Und wenn nicht begrifflich, so sind funktionell noch heute ähnliche Phänomene unübersehbar. So finden sich wochentags, wenn der Landeshauptmann der Autonomen Provinz Bozen-Südtirol ab 6.30 Uhr täglich seine »Sprechstunden « abhält, zahlreiche Menschen auch aus abgelegenen Landesteilen vor dem Landhaus ein, um höchstpersönlich die verschiedensten Anliegen direkt beim populären »Landesvater " zu deponieren. Diese werden stets bearbeitet, ohne dass die Geschäftsverteilung zwischen den Mitgliedern der Landesregierung streng eingehalten würde.

Supplikationen über Supplikationen - wo bleiben die im Titel angekündigten Gravamina? Es ist dies eine Frage, die sich der Rezensent ebenfalls gestellt hat, finden sich die Gravamina (also im institutionalisierten Kontext von Land- tagen vorgebrachte Beschwerden der Landstände) doch nur vergleichsweise am Rande thematisiert. Was vielleicht etwas irritierend sein mag, dem aber durchaus Positives abgewonnen werden kann: Nur allzu viele Sammelbände sind dem Risiko einer gewissen inhaltlichen Inhomogenität ausgesetzt, was hier kaum der Fall ist.

Die wissenschaftliche Beschäftigung mit Suppliken ist seit einigen Jahren jedenfalls en vogue. Rechtsgeschichte, Verwaltungsgeschichte, Forschungen zur "guten Policey ", Historische Kriminalitätsforschung, Gesetzgebungsgeschichte, Alltags- und Sozialgeschichte: Die inhaltliche Bandbreite des Quellentypus "Supplikation « macht diesen zu einem lohnenden Untersuchungsgegenstand für eine Vielzahl von (Teil-) Disziplinen. Dies verdeutlichen bereits die einführenden Beiträge der Herausgeber Andreas Würgler und Cecilia Nubola. ${ }^{\text {I }}$ Würgler liefert einen Abriss der Erträgnisse der deutschsprachigen Frühneuzeitforschung zu den Themenbereichen » Gravamina « und »Supplikationen ", während Nubola eine Zusammenschau über die Bedeutung und Funktion der Supplikationen in den italienischen Staaten vom I5. bis zum I 8. Jahrhundert erstellt. Anschließend werden von deutsch- und italienischsprachigen Forschern Fallstudien vorgelegt, die in drei Teile gegliedert sind und das breite Spektrum an Fragestellungen erkennen lassen, in deren Rahmen Supplikationen ausgewertet werden können. Der erste Teil mit Artikeln von Gian Maria Varanini, Nadia Covini, André Holenstein und Marina Garbellotti thematisiert die Funktion von Supplikationen in der Verwaltungspraxis, während die Beiträge des zweiten Teiles (Karl Härter, Irene Fosi und Renate Blickle) ihre zentrale Rolle für die Aushandlung von Strafen im Kontext der frühneuzeitlichen Strafjustiz aufzeigen. Der dritte Teil (Diego Quaglioni, Christian

\footnotetext{
I Der einleitende Beitrag von

ANDREAS Würgler stellt die überarbeitete Fassung eines Beitrags dar, der bereits 1999 in italienischer Sprache erschienen ist: Suppliche e 'gravamina< nella prima età moderna: la storiografia di lingua tedesca, in: Annali dell'Istituto storico italo-germanico in Trento 25 (I999) 5I 5-546.
} 
Zendri, Angela De Benedictis, Laura Turchi, Harriet Rudolph) beleuchtet den Zusammenhang von Widerstand und Aufbegehren einerseits und Suppliken andererseits. Abschließend versucht Giorgio Politi eine Gesamtwürdigung der »Gravamina « als einem der Charakteristika der (west)europäischen Geschichte. Der Beitrag von De Benedictis, der den Leser in das Frankreich der Religionskriege führt, erweitert dabei den Untersuchungsraum über den deutsch- und italienischsprachigen Bereich hinaus. Der dritte Teil umfasst überdies zwei Beiträge, die inhaltlich etwas aus dem Rahmen fallen. Die Untersuchungen von Quaglioni über die Strafbarkeit von Körperschaften nach der gemeinrechtlichen Lehre sowie von Zendri über das Verhältnis von Gesetz und Gewohnheitsrecht bei Ulrich Zasius (I46I-I 535) sind zwar instruktiv, der Konnex mit dem Generalthema »Gravamina und Supplikationen « erschließt sich jedoch nur auf ausgedehnten Umwegen.

In ihrer Gesamtheit unterstreichen die Beiträge die fundamentale Bedeutung von Supplikationen für die frühneuzeitliche Verwaltungsund Justizpraxis und für die Bewältigung respektive Kanalisierung von Konflikten in der ständischen Gesellschaft. Sie verdeutlichen, dass Herrschaft im Ancien Régime ein kommunikativer Prozess ständigen Aushandelns und Vereinbarens zwischen Obrigkeiten und Untertanen war. ${ }^{2}$ Die Analysen der deutschsprachigen Beiträge konzentrieren sich dabei auf die territoriale Ebene: Holenstein bezieht sich auf Baden-Durlach, Härter auf Kurmainz, Blickle auf Bayern und Rudolph auf Osnabrück. Die italienischen Beiträge sind breiter gestreut: Der Untersuchungsraum reicht dabei von einer Tiroler landesfürstlichen Kleinstadt (Rovereto) über italienische Kommunen (Verona, Mailand) bis hin zum Kirchenstaat der Barockzeit. Zeitlich wird ebenfalls ein breiteres Spektrum abgedeckt, da auch das Spätmittelalter Beachtung findet. Dessen geringe Beachtung durch die deutschsprachigen Untersuchungen mag durch die spärlichere archivalische Überlieferungslage nördlich der Alpen bedingt sein.

In einigen Punkten scheint jedoch noch Diskussionsbedarf zu bestehen bzw. werden weiterführende Fragen aufgeworfen. Dies trifft in besonderem Maße auf den Beitrag von André Holenstein zu (»>Ad supplicandum verweisen<. Supplikationen, Dispensationen und die Policeygesetzgebung im Staat des Ancien Régime", I67-210). Wäre hier nicht eine genauere Differenzierung zwischen der Möglichkeit individueller Dispensationen von der Geltung einer Policeynorm einerseits und der Erteilung von Bewilligungen aufgrund einer Policeynorm andererseits wünschenswert? Salopp formuliert: "Dispensabel ist alles «, dispensare gehört wie leges ferre et interpretari zu den Kernbestandteilen der landesherrlichen potestas legislatoria, und natürlich wurde die Möglichkeit der Dispenserteilung bei Erlass und Vollzug einer Policeynorm von der Verwaltung mitbedacht. Und doch dürfte es ein fundamentaler Unterschied sein, ob eine (Verbots-)Norm »nur « die allgemeine Möglichkeit einer Dispenserteilung in sich trägt oder ob von vornherein ein bewilligungspflichtiges Vorhaben statuiert wird (wobei sich die Voraussetzungen für die Erteilung einer Bewilligung entweder aus der Norm selbst oder konkretisierenden Reskripten ergeben oder vom Historiker aus der Vollzugspraxis erschlossen werden können). Zielsetzung dürfte in diesen Fällen eine andere sein: Die verfahrenseinleitenden Supplikationen erfüllen hier zwar auch die Funktion einer "administrativen Überwachung des Gesetzesvollzugs « (I90) oder der Information der Behörde über die Reichweite einer

2 Vgl. auch Markus MeumanN, Ralf Pröve (Hg.), Herrschaft in der Frühen Neuzeit. Umrisse eines dynamisch-kommunikativen Prozesses (Herrschaft und soziale Systeme in der Frühen Neuzeit, Bd. 2), Münster 2004 . 
Policeynorm. Im Zentrum steht der jeweilige Schutzzweck der Policeynorm, der materienspezifisch zu bestimmen ist. Hierzu nur zwei Beispiele: Die Bewilligung zum Eintritt in fremde Kriegsdienste wird versagt werden, wenn es sich beim zukünftigen Arbeitgeber um eine (potentiell) feindliche Macht handelt. Handelt es sich dagegen um eine befreundete Macht, kann die Erlaubnis erteilt werden, wobei verschiedene Ordnungsziele eine Rolle spielen können: diplomatische Rücksichten, die Bevölkerungs- und Wirtschaftsentwicklung des eigenen Landes oder allenfalls geplante eigene Söldnerrekrutierungen. Die Überwachung des Liegenschaftsverkehrs von fürstlichen Bediensteten empfiehlt sich, da mit einem entsprechenden Grundstückskauf unter Umständen Unterschiede in der Besteuerung oder eine Verlagerung des Gerichtsstandes weg vom forum rei sitae einhergehen können. Es können natürlich Verbindungen zwischen Dispensgesuchen und der Einführung von Bewilligungspflichten bestehen: Härter weist nach (27I-272), dass Verbote von Festveranstaltungen an Sonn- und Feiertagen eine Vielzahl von Dispensgesuchen und Normübertretungen nach sich zogen, so dass schließlich seit der Mitte des I 8. Jahrhunderts Strafen und Dispensgelder zu einer "Vergnügungssteuer " ausgebaut wurden. Holenstein betont ferner die große Rolle der "Gnade « bei der Bewilligungserteilung im Baden des I8. Jahrhunderts (vgl. bes. I9I-I92). Dies ist insofern überraschend, als Härter, der nicht nur die " peinliche " Strafgerichtsbarkeit, sondern auch die Policeynormen in seine Untersuchung einbezieht, aufzeigt, dass das Supplizieren in diesem Bereich keineswegs ausschließlich auf die Thematik "Gnade « reduziert werden kann. Holenstein weist dagegen der »Gnade « für Dispensationen und Bewilligungen im Policeyrecht eine zentrale Bedeutung zu, wenngleich er kon- zediert, dass dieses »Gnadenrecht « im Absolutismus "teilweise seine charismatischen Merkmale« (I95) verloren habe. Haben wir es mit einer Besonderheit der baden-durlachischen Verwaltung zu tun? Woran lässt sich die Relevanz der Gnade « in der administrativen Praxis festmachen? Der zentralen Kategorie »Gnade« im Policeyrecht des I 8. Jahrhunderts stellt Holenstein dabei das moderne Verwaltungsrecht gegenüber, für das in Anbetracht des Legalitätsprinzips »die Gnade eine systemwidrige Erscheinung darstellt" (I92). Hier könnte der Jurist nachhaken, zumindest der österreichische: Zwar gilt heute natürlich das sehr strenge Legalitätsprinzip des Art. I8 Bundes-Verfassungsgesetz - aber nur im (verhältnismäßig schmalen) Bereich der hoheitlichen Verwaltung, nicht dagegen im umfassenden Bereich der so genannten »Privatwirtschaftsverwaltung «. Hier mögen Selbstbindungsgesetze oder interne Richtlinien (so genannte »Verwaltungsverordnungen «) das Handeln der Verwaltung vorhersehbar machen; es besteht jedoch kein Rechtsanspruch des Bürgers auf ein bestimmtes Tätigwerden, noch kann eine Entscheidung von einer übergeordneten Instanz überprüft werden. Ob jemand vom Amt einer Landesregierung einen Druckkostenzuschuss für eine Dissertation erhält, ist letztlich in das Ermessen des Sachbearbeiters gestellt. Ist das auch auf die Kategorie »Gnade « zu reduzieren? Könnte man gerade für das I 8. Jahrhundert nicht auch der Sicht von Hans Maier und Reiner Schulze folgen, wonach gerade die zunehmende Policeygesetzgebung langfristig zu einer stärkeren Bindung der Verwaltung durch das Recht geführt habe?3 Wurde so nicht die "Gnade " kanalisiert? Hier eröffnen sich noch interessante Diskussionsfelder.

Diskutabel erscheint ferner die Zurechnung der (anonymen) Anzeigen zur Quellengattung

3 Vgl. zum Überblick AlF LÜDTKE, Herbert Reinke, Crime, Police and »The Good Order «. Germany, in: Crime History and Histories of Crime. Studies in the Historiography of Crime and Criminal Justice in Modern History, hg. von Clive Emsley, Louis A. Knafla, London I996, I09-I37, hier: II $5 \mathrm{f}$. 
der "Supplikationen ", wie dies mehrere Autoren postulieren (vgl. Würgler, 48 , der die Denunziation als "Sonderfall der Supplik « bezeichnet; ähnlich Nubola, 67-69 und Härter, 255-258). Gerade in den Fällen von "Justiznutzung ", in denen der Obrigkeit deviantes Verhalten von einer namentlich genannten Person in Briefform gemeldet wurde, liegt formal tatsächlich häufig eine Supplik vor (Verwendung verbaler Demutsbezeugungen gegenüber dem Adressaten, Schilderung der Vorfälle, die zu dessen Anrufung als letztem Ausweg führten sowie Bitte um Abhilfe). Bei anonymen Anzeigen ist regelmäßig nicht einmal mehr diese formal-strukturelle Ähnlichkeit mit der Supplikation gegeben. Und inhaltlich ist wohl eine grundsätzliche Differenz zwischen der Anzeige, mag diese anonym sein oder nicht, und der Supplikation festzustellen. Der Supplikant will unabhängig vom Kontext, in dem die Bitte artikuliert wird, stets etwas für jemanden erreichen: für sich selbst oder (wie es Renate Blickle anhand der Interzession thematisiert) für eine nahe stehende Person. Die Anzeige dagegen richtet sich primär gegen jemanden, erst in zweiter Hinsicht ergibt sich ein Zusatznutzen für den Anzeiger (so die Entlastung vom gewalttätigen, sliederlichen< Ehemann, die Disziplinierung des >unnützen`Sohnes, die Absetzung eines korrupten Amtsträgers). Funktional mögen gewisse Ähnlichkeiten gegeben sein, wie die Information für die Obrigkeit; die Unterschiede treten jedoch klar hervor. Heutzutage käme niemand auf die Idee, die Anzeige gegen einen Dritten wegen einer Verwaltungsübertretung und die Bitte um einen Druckkostenzuschuss für eine Dissertation in einen Topf zu werfen - für die Frühe Neuzeit sind vergleichbare Bedenken geringer ausgeprägt.

Es ist das große Verdienst des Sammelbandes, dem Leser nicht nur die vielfältigen Auswertungsmöglichkeiten der Quellengattung "Supplikation « exemplarisch und auf hohem Niveau vor Augen zu führen, sondern zudem den Blick auf die italienische Forschungslandschaft zu erweitern.

Martin Schennach

\section{Key Ideas in Islamic Law*}

\section{Introduction}

History is essential for understanding, and in today's difficult climate there are few areas which are in greater need of understanding, particularly for non-Muslims, than Islamic law. Yet, in Chibli Mallat's words: "A history of Islamic law, let alone of Middle Eastern law, is yet to be written. " ${ }^{\mathbf{I}}$

Indeed, as Wael Hallaq points out in his introduction, ${ }^{2}$ our level of knowledge of the most important part of the history of Islamic law, its origins and early evolution, is surprisingly low, despite considerable improvements in recent years. The difficulty is increased by the lack of a general monograph, as the recent scholarship which has cast new light on some areas and disproved earlier ideas in others, making "classic" works such as those by Coulson and Schacht largely out of date and misleading in many respects, is mostly scattered in journal articles and book chapters. Those monographs

\footnotetext{
Wael B. Hallaq, The Origins and Evolution of Islamic Law, Cambridge, New York: Cambridge University Press 2004, ix, 246 pp., ISBN 0-52 I-80332-2; this review has benefited from that by Professor William BallanTYNE, , Wael Hallaq: The Origins and Evolution of Islamic Law , in: Journal of Comparative Law I (2006) 208.
}

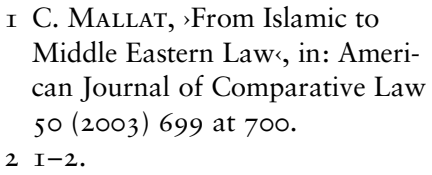

